\title{
Budget Impact Analysis of Using Dihydroartemisinin- Piperaquine to Treat Uncomplicated Malaria in Children in Tanzania
}

\author{
Amani Thomas Mori ${ }^{1,2} \cdot$ Ole Frithjof Norheim ${ }^{1,3} \cdot$ Bjarne Robberstad $^{1,3}$
}

Published online: 31 October 2015

(c) The Author(s) 2015. This article is published with open access at Springerlink.com

\begin{abstract}
Background and Objective Dihydroartemisinin-piperaquine $(\mathrm{DhP})$ is a very cost effective anti-malarial drug. The aim of this study was to predict the budget impact of using DhP as a first- or second-line drug to treat uncomplicated malaria in children in Tanzania.

Methods A dynamic Markov decision model was developed based on clinical and epidemiological data to estimate annual cases of malaria in children aged under 5 years. The model was used to predict the budget impact of introducing $\mathrm{DhP}$ as the first- or second-line anti-malarial drug, from the perspective of the National Malaria Control Program in 2014; thus, only the cost of drugs and diagnostics were considered. Probabilistic sensitivity analysis was performed to explore overall uncertainties in input parameters. Results The model predicts that the policy that uses artemether-lumefantrine (AL) and $\mathrm{DhP}$ as the first- and second-line drugs $(\mathrm{AL}+\mathrm{DhP})$, respectively, will save about $\$$ US64,423 per year, while achieving a $3 \%$ reduction in the number of malaria cases, compared with that of
\end{abstract}

Amani Thomas Mori

pax_amani@yahoo.com

Ole Frithjof Norheim

ole.norheim@uib.no

Bjarne Robberstad

bjarne.robberstad@uib.no

1 Centre for International Health, Department of Global Public Health and Primary Care, University of Bergen,

P.O. Box 7804, 5020 Bergen, Norway

2 Muhimbili University of Health and Allied Sciences, P.O. Box 65001, 11103 Dar es Salaam, Tanzania

3 Centre for Intervention Science in Maternal and Child Health, University of Bergen, P.O. Box 7804, 5020 Bergen, Norway
$\mathrm{AL}+$ quinine. However, the policy that uses DhP as the first-line drug $(\mathrm{DhP}+\mathrm{AL})$ will consume an additional \$US780,180 per year, while achieving a further $5 \%$ reduction in the number of malaria cases, compared with that of $\mathrm{AL}+\mathrm{DhP}$.

Conclusion The use of DhP as the second-line drug to treat uncomplicated malaria in children in Tanzania is slightly cost saving. However, the policy that uses DhP as the first-line drug is somewhat more expensive but with more health benefits.

\section{Key Points for Decision Makers}

Understanding the financial burden that will be imposed by a new health technology on the health system is important for planning and budgeting.

Dihydroartemisinin-piperaquine is relatively more expensive than artemether-lumefantrine but has a greater potential to reduce the burden of Plasmodium falciparum malaria when used as the first-line drug to treat uncomplicated malaria.

\section{Introduction}

Malaria is an infectious disease, usually of short duration; there were an estimated 198 million cases of malaria (range 124-283 million) and an estimated 584,000 deaths (range 367,000-755,000) globally in 2013 [1]. In Tanzania, malaria is responsible for about one-tenth of all outpatient 
fevers in children [2]. Most malaria infections in sub-Saharan Africa are caused by Plasmodium falciparum, and without adequate treatment, the disease can progress rapidly to life-threatening severe malaria. There is a lot of controversy surrounding the precise burden of malaria in African countries. In 2010, malaria deaths in Tanzania among children aged under 5 years were estimated to range between 10,928 and 49,663 [3]. In 2013, WHO estimates showed that there were about 14.6 million cases of suspected malaria in the country, which places Tanzania among 18 countries with the highest burden of malaria in the sub-Saharan African region [1].

Tanzania has repeatedly changed its malaria treatment guidelines in response to $P$. falciparum resistance to antimalarial drugs. In 2001, chloroquine was replaced with sulfadoxine-pyrimethamine (SP) as the first-line drug to treat uncomplicated malaria. In 2007, SP was replaced with artemether-lumefantrine (AL), while quinine was a recommended second-line drug (AL + quinine) [4]. In 2014, quinine was replaced with dihydroartemisinin-piperaquine $(\mathrm{DhP})$ as the second-line drug $(\mathrm{AL}+\mathrm{DhP})$ [5]. $\mathrm{AL}$ and $\mathrm{DhP}$ are two of the five artemisinin-based combination therapies (ACTs) recommended by the WHO to treat uncomplicated malaria [6].

Recent research evidence indicates that DhP may be a better first-line drug to treat uncomplicated malaria than AL because it is more effective [7] and offers a prolonged post-treatment prophylaxis, which reduces the risk of recurrent malaria infections $[8,9]$. In addition, DhP has a simple once-a-day dosage regimen which enhances adherence to treatment [10]. Economic evaluation studies have also indicated that $\mathrm{DhP}$ is more cost effective than $\mathrm{AL}$ when used as the first-line drug; hence, it represents better value for money [8, 11, 12]. Mori et al. [12] showed that DhP was more cost effective than AL from a providers' perspective, with an incremental cost-effectiveness ratio of \$US12.40 per disability-adjusted life-year (DALY) averted in a Tanzanian setting, based on a valuation performed in December 2012 [12].

Cost-effectiveness analysis compares costs and outcomes of competing interventions, and is an important criterion for priority-setting decisions. However, such analyses fail to report information that can be used to assess whether costeffective interventions are also affordable to the purchaser. Budget impact analysis (BIA), which addresses the issue of affordability, is therefore increasingly used to complement cost-effectiveness analyses. BIA estimates the financial consequences of adopting a new drug within a specific healthcare setting to guide formulary listing decisions [13]. This study aims to predict the budget impact of adopting DhP as the first- or second-line drug to treat uncomplicated malaria in children in Tanzania compared with a previous policy composed of $\mathrm{AL}$ and quinine.

\section{Methods}

\subsection{Analytical Framework}

The BIA was conducted using a dynamic Markov cohort model with four mutually exclusive health states: "well", "uncomplicated malaria", "severe malaria", and "death" as an absorbing health state. The model is a modified version of the one used to compare the cost effectiveness of $\mathrm{AL}$ and DhP when used as the first-line drugs in Tanzania [12]. While the original model was closed, this is an open model, which allows entrance of new members through births while others exit after reaching the age of more than 5 years over the 1-year time horizon of the model (Fig. 1). The cohort begins in a "well" state and then transits to other health states in 1-week cycles based on risk factors for malaria, access to healthcare, and the effectiveness of anti-malarial drugs.

The analysis was performed from the perspective of the National Malaria Control Program and has a duration of 1 year. A probabilistic sensitivity analysis (PSA) was conducted using Monte-Carlo simulations to assess the robustness of the model considering the uncertainty associated with the input parameters. The analysis was run using TreeAge Pro 2015 software (TreeAge Software, Inc., Williamstown, PA, USA). The study adheres to the BIA guidelines regarding principles of good practice from the International Society for Pharmacoeconomics and Outcome Research (ISPOR) [13].

\subsection{Intervention Mix}

Two competing treatment policies for uncomplicated malaria, each consisting of AL and DhP as first- or secondline drugs, were evaluated against a reference policy of $\mathrm{AL}+$ quinine. A regimen consisting of a 3-day dosage of parenteral quinine, followed by an oral dose of the first-line drug, was employed as a standard treatment for severe malaria in all policies. The treatment policies involved are:

1. $\mathrm{AL}+$ quinine: this is a reference policy, which uses $\mathrm{AL}$ and quinine as first- and second-line drugs, respectively. This policy was launched in 2006 and followed until it was replaced in 2014 by $\mathrm{AL}+\mathrm{DhP}$ [4].

2. $\mathrm{AL}+\mathrm{DhP}$ : this policy option substitutes quinine with $\mathrm{DhP}$ as the second-line drug in the reference policy and is consistent with the policy change of 2014 in the country [5]. This treatment policy is also consistent with WHO recommendations regarding the treatment of uncomplicated malaria [6].

3. $\mathrm{DhP}+\mathrm{AL}$ : this policy uses $\mathrm{DhP}$ as the first-line drug and $\mathrm{AL}$ as the second-line drug. This policy would 
Fig. 1 State transition diagram of the model

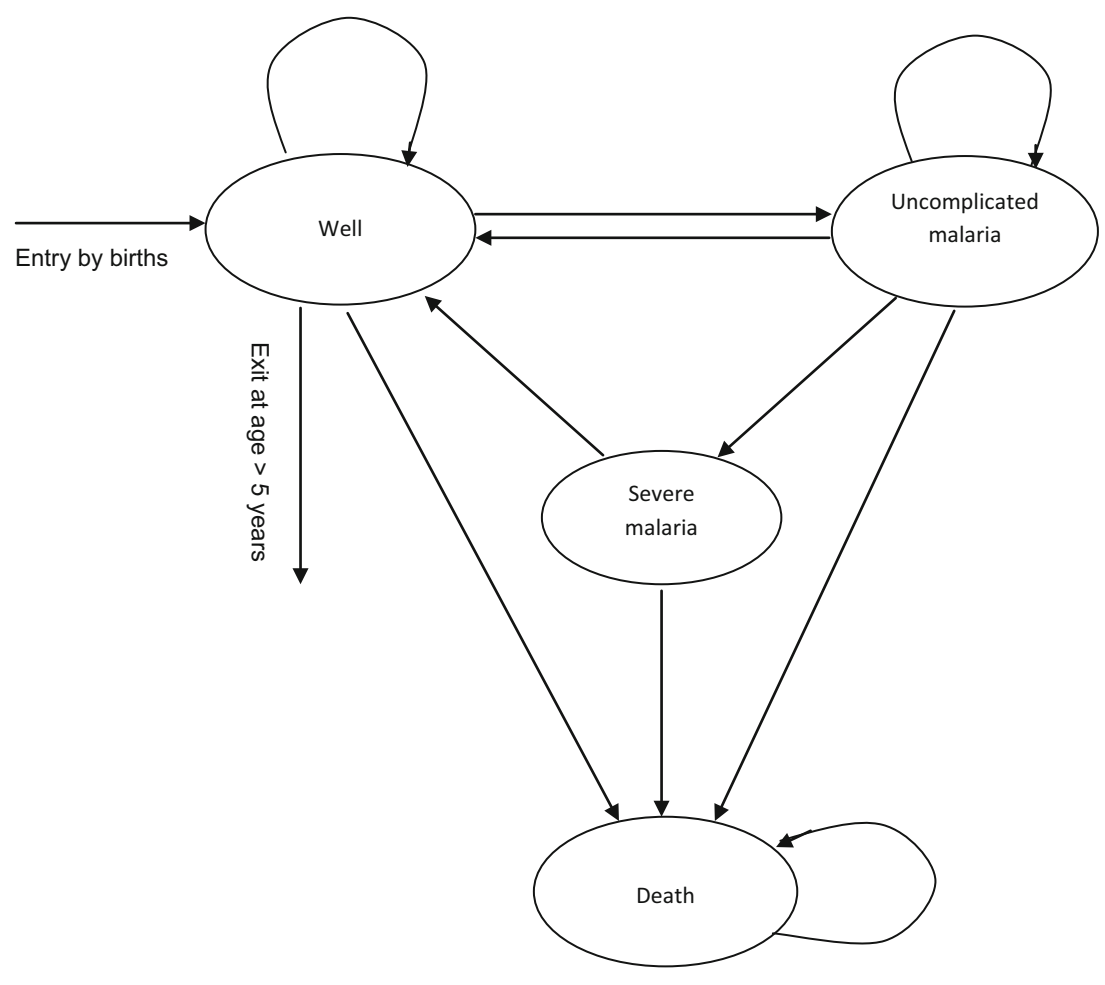

exploit the benefits of DhP, which include relatively higher efficacy and compliance rates. It is also consistent with WHO recommendations regarding the treatment of uncomplicated malaria [6].

\subsection{Patient Population}

The study was conducted in a sub-population of 7,273,832 children aged under 5 years in Tanzania [14]. Children are most vulnerable to malaria and account for more than twothirds of all malaria deaths [1]. Estimates of the size of the eligible population was based on a 2 -week prevalence of fevers of about $20 \%$ [15], of which about $10.5 \%$ have been attributed to malaria infections [2]. The cohort grows at a rate of about 8466 children per week, which is the difference between the birth and aging rate $[14,16]$. The all-cause mortality rate of 81 per 1000 live births for children aged under 5 years [17] was adjusted downward by $11 \%$ to account for deaths due to causes other than malaria [18]. Progression to severe malaria and survival rates in each arm depend on the effectiveness of the firstand second-line drugs, which in this study are influenced by differences in compliance rates.

\subsection{Characteristics of Anti-Malarial Drugs}

A large head-to-head, multicenter randomized clinical trial indicated that DhP has a cure rate of 97.3 versus $95.5 \%$ for
AL, based on an intention-to-treat analysis. The administration of drugs in this trial was directly observed and children stayed at the facilities long enough to check for any vomiting, which implies perfect adherence to treatment [19]. DhP is administered once daily while AL should be taken twice daily together with fat-rich meals, both for 3 consecutive days. Compliance rates for $\mathrm{AL}$ and $\mathrm{DhP}$ range from 60 to 80 and 70 to $90 \%$, respectively [10, 20].

Quinine has been used as a second-line drug for the management of uncomplicated malaria in endemic countries for many years, mostly due to the lack of an appropriate alternative drug. The major limitations of quinine are its long, three times daily dosage regimen, which extends to 7 days, and cinchonism adverse effects. In a nested clinical trial, quinine used as a second-line drug to treat recurrent malaria infection in children in Uganda showed cure rates ranging from 88 to $98 \%$ in a head-to-head comparison with AL and DhP [21]. An assumed compliance rate varying from 40 to $60 \%$ was used in this study, based on a clinical trial and one other economic study [22, 23].

Patient compliance to treatment is a primary determinant of therapeutic outcomes. Poor compliance to antimalarial drugs increases the chances of treatment failure and, more importantly, drug resistance. However, in economic modeling studies it is commonly assumed that $10-20 \%$ of non-compliers to multi-dosage anti-malarial drugs such as the ones considered in this study may 
experience spontaneous recovery $[24,25]$. Therefore, in order to predict cure rates, we combined efficacy rates from trial settings and adherence rates observed in routine clinical practice using the following formula (Eq. 1):

Cure rate $=E_{0} C+E_{\mathrm{nc}}(1-C)$

where $E_{0}$ is efficacy, $C$ is the compliance rate, and $E_{\text {nc }}$ is the proportion of non-compliers who may experience spontaneous recovery.

\subsection{Assumptions}

The model was based on the following simplifying assumptions:

- The policy implementation has reached a steady state.

- There is no asymptomatic malaria state; hence, all cases of severe malaria are due to progression from uncomplicated malaria.

- Uncomplicated malaria is not fatal and all positive cases in the public facilities are prescribed the recommended first-line drug.

- The second-line drug ACT will be restricted in the public sector, and when it is out of stock patients will be given the first-line drug.

\subsection{Care Seeking for Diagnosis and Treatment of Malaria}

Care-seeking behavior for the treatment of malaria is very complex and is influenced by many factors, such as perceived severity of the disease, proximity to the facility, availability of medicine, and the ability to pay for health services $[26,27]$. In this study, caregivers are modeled to seek care for their febrile children from three main sources: the public sector, which also includes non-profit faithbased facilities; the private sector, which is composed of accredited drug shops and pharmacies; and informal sources. A recent national HIV/AIDS and Malaria Indicator Survey reported that between 77 and $81 \%$ of caregivers sought care from formal health facilities [15]. Between 50 and $70 \%$ of these formal facilities are composed of public facilities, and the remainder include private-sector pharmacies and drug shops, which are scattered all over the country [28].

Between 50 and $70 \%$ of all febrile children who visit public facilities are treated based on malaria Rapid Diagnostic Test (mRDT) results and the rest are treated based on presumptive diagnosis $[29,30]$. Non-adherence by health workers to a negative mRDT was estimated to vary from 7 to $14 \%$ [31, 32]. Test results were based on Bayesian calculations, with a prior positive test probability of $10.5 \%$, and sensitivity and specificity (range) of the
mRDT of $95.4 \% \quad(94.2-96.6 \%)$ and $95.9 \%$ (94.8-97.0\%), respectively [33]. The sensitivity and specificity (range) of presumptive diagnosis were set at $30 \%(20-40 \%)$ and $90 \%(80-100 \%)$, respectively [34].

Availability of the first-line drug in public facilities varies from 40 to $80 \%$ [35], and when it is out of stock between 40 and $60 \%$ of patients will be able to access it from private-sector drug shops and pharmacies [36]. The rest may purchase non-recommended drugs, with cure rates ranging between 10 and $60 \%$ [37]. If a child still has uncomplicated malaria after the initial treatment, we assume the caregiver will choose to return to the public facility, where the second-line drug will be prescribed, with availability of $80-100 \%$ for quinine (i.e., because it is relatively cheap) and 40-60\% for ACTs.

Private-sector drug shops and pharmacies are an important first point of care for patients with suspected malaria infections in Tanzania. However, diagnostic tests are usually unavailable; hence, treatments are based on symptoms alone. A majority of these premises stock ACTs, following the implementation of the Affordable Medicines Facility-malaria (AMFm) program [38]. Considering that the major incentive of these premises is to maximize sales, we assume that $60-80 \%$ of febrile children receive antimalarial drugs [39], of which about $40-60 \%$ are a recommended first-line drug [40]. To capture the complexity of care seeking, we assumed that half of the patients who fail to respond to treatments will choose to shift to the public health facilities and the rest will remain in the private sector.

In Tanzania, only formal healthcare facilities, including the private-sector accredited drug shops and pharmacies, are authorized to stock anti-malarial drugs. Therefore, all treatments sought from informal sources such as markets, local shops, and traditional healers are considered to be ineffective. However, we assumed that $10-20 \%$ of these cases may experience spontaneous recovery [12]. Those who continue to suffer from uncomplicated malaria will shift to the public healthcare facilities for further treatment.

Between 3 and $7 \%$ of untreated malaria cases, or those who experience treatment failure, progress to severe malaria [25, 41]. Between 80 and $100 \%$ of severe cases of malaria have been estimated to have access to adequate inpatient care [42], which reduces the case-fatality rate, ranging between 45 and $80 \%$ [43], to about $10.9 \%$ [44].

\subsection{Resource Use and Costs}

A costing study conducted at an urban district-level hospital exists and this has been reported elsewhere [12]. However, due to its lack of representative cost data for personnel and other items, the current BIA study focuses on expenditures incurred on ACTs, mRDTs, and other 
drugs used to manage the associated co-morbidities. This is more relevant to the National Malaria Control Program, which is responsible for the development of malaria policies and budgeting for ACTs and mRDTs, which are largely funded by donors. For uncomplicated malaria, the drugs included are the recommended first- and second-line anti-malarials. We included SP also to represent the use of non-recommended drugs, especially from the private-sector premises [15, 28]. Antipyretics such as paracetamol were omitted because they are relatively cheap and hence unlikely to have significant budgetary implications. For severe malaria, the cost of a standard regimen consisting of parenteral quinine, diclofenac, diazepam, hematinics, and a first-line ACT was included [5].

The Global Fund's reference prices negotiated with the manufacturers of ACTs for children aged under 5 years in endemic countries were used [45], while for SP a buyer price (range) of \$US0.32 (\$US0.25-0.38) from the International Drug Price Indicator Guide was used [46]. Prices of ACTs were inflated by $20 \%$ to account for program costs, i.e., $10 \%$ freight and insurance costs [46] and $10 \%$ as estimated local distribution costs. Prices of other drugs were taken from the price catalogue of the Medical Stores Department (MSD) [47]. Prices of mRDTs have decreased recently due to price competition; hence, a unit price (range) of \$US0.45 (\$US0.36-0.55) was used [30]. An exchange rate of 1670 Tanzanian shillings to \$US1 for the year 2014 was used to convert local MSD prices into US dollars [48].

\subsection{Uncertainty}

Probability distributions were employed to incorporate uncertainties in the parameters used in the model (Table 1). Beta distributions were used for probability parameters to limit their possible values to the interval $0-1$, while costs were constrained between 0 to positive infinity by gamma distributions. Normal distributions were used to describe estimated population size, birth rates, and compliance rates of different anti-malarial drugs. Overall uncertainty in the parameters was propagated in the model by running a PSA based on a Monte-Carlo simulation with 10,000 iterations. One-way sensitivity analysis was used to test how variations in key parameters, including addition of hospitalization costs, may potentially influence the results.

\section{Results}

\subsection{Model Validation}

The model was validated using the reference policy $(\mathrm{AL}+$ quinine) to determine whether the predicted health outcomes from the simulations correspond with the figures reported in the literature. An estimated 10-12 million cases of uncomplicated malaria occur in Tanzania annually, of which about two-thirds are in children aged under 5 years [30]. Our model prediction of 7,510,727 cases is therefore consistent with the implied range of $6.7-8.0$ million cases. The model predicted 173,600 cases of severe malaria among children aged under 5 years, which is also about two-thirds of all severe cases based on the WHO World Malaria Report 2013 [50]. The predicted total number of deaths in children aged under 5 years was 134,028 , which is within the range of $123,100-186,700$ [51]. The predicted number of malaria-attributable deaths was 26,973 , which is within the reported range of 10,928-49,663 for children aged under 5 years in Tanzania [3].

\subsection{Budget Impact Analysis}

The model predicts that the treatment policy of $\mathrm{AL}+\mathrm{DhP}$ will save about \$US64,423 (0.3\%) per year, compared with that of $\mathrm{AL}+$ quinine, and the policy of $\mathrm{DhP}+\mathrm{AL}$ will consume an additional \$US780,180 (3.8\%) per year compared with that of AL $+\mathrm{DhP}$. These represent changes in the budget for drugs and mRDTs only (Table 2). It is interesting to note that $\mathrm{DhP}+\mathrm{AL}$ has the highest drug costs but the lowest costs for mRDT compared with other policies. The reason for this difference in cost is that the higher effectiveness rate of $\mathrm{DhP}$ as a first-line drug reduces episodes of malaria and hence the requirement for mRDT.

\subsection{Impact on Health Outcomes}

Table 3 shows the estimated change in overall annual health outcomes, as recommended by the new guidelines for reporting BIA analysis [13], for the two malaria treatment policies. The model predicts that the policy of $\mathrm{AL}+\mathrm{DhP}$ has the potential to reduce the number of malaria cases and deaths by 248,437 (3\%) and 1954 (7.2\%), respectively, compared with the reference policy of $\mathrm{AL}+$ quinine. However, the policy of $\mathrm{DhP}+\mathrm{AL}$, which is more expensive, has the potential to reduce the number of malaria cases and deaths further by 364,517 (5\%) and $2868(11.5 \%)$, respectively, compared with the policy of $\mathrm{AL}+\mathrm{DhP}$.

\subsection{Sensitivity Analyses}

The policy of $\mathrm{AL}+$ quinine has already been replaced with that of $\mathrm{AL}+\mathrm{DhP}$, and since it is probably not very attractive to revert to quinine-based therapy, policy makers will be more interested in a comparison of $\mathrm{DhP}+\mathrm{AL}$ versus $\mathrm{AL}+\mathrm{DhP}$. This is presented in the Tornado 
Table 1 Parameters used in the model and their distributions

\begin{tabular}{|c|c|c|c|}
\hline Parameters & Estimates & Distributions & Sources \\
\hline Cohort size of children aged under 5 years & $7,273,832 \pm 20 \%$ & Normal & {$[14]$} \\
\hline Weekly cohort growth rate & $8466 \pm 20 \%$ & Normal & {$[14,16]$} \\
\hline Under-five mortality rate per 1000 live births & $81(72-90 \%)$ & Beta & {$[17]$} \\
\hline Malaria-attributed deaths in children aged under 5 years & $11 \%$ & Point estimate & {$[18]$} \\
\hline 2-Week incidences of fever episodes per child & $0.20 \pm 20 \%$ & Beta & [15] \\
\hline Percentage of febrile episodes attributed to malaria & $10.5 \pm 20 \%$ & Beta & {$[2]$} \\
\hline Case fatality rate of untreated severe malaria & $60(45-80 \%)$ & Beta & [43] \\
\hline Case fatality rate of treated severe malaria & $10.9 \%$ & Beta & [49] \\
\hline Early treatment failure leads to severe malaria & $5(3-7 \%)$ & Beta & [41] \\
\hline Untreated malaria becomes severe & $5(3-7 \%)$ & Beta & {$[25]$} \\
\hline Probability of care seeking in formal facilities & $79(77-81 \%)$ & Beta & {$[15]$} \\
\hline Percentage of formal facilities belonging to public sector & $60(50-70 \%)$ & Beta & {$[28]$} \\
\hline Percentage of severe cases with access to inpatient care & $90(80-100 \%)$ & Beta & [42] \\
\hline Access to first-line drugs in public facilities & $60(40-80 \%)$ & Beta & {$[35]$} \\
\hline Access to second-line drugs in public facilities & $50(40-60 \%)$ & Beta & Assumption \\
\hline Percentage of children given anti-malarials in private facilities & $70(60-80 \%)$ & Beta & [39] \\
\hline Percentage of anti-malarials that are ACT in private facilities ${ }^{\mathrm{a}}$ & $50(40-60 \%)$ & Beta & {$[36,40]$} \\
\hline Efficacy of DhP & $97.3(94.9-99.7 \%)$ & Beta & [19] \\
\hline Efficacy of AL & $95.5(93.1-97.9 \%)$ & Beta & [19] \\
\hline Efficacy of quinine & $93.0(88.0-98.0 \%)$ & Beta & {$[21]$} \\
\hline Compliance with AL & $70(60-80 \%)$ & Normal & {$[10,20]$} \\
\hline Compliance with DhP & $80(70-90 \%)$ & Normal & {$[10,20]$} \\
\hline Compliance with quinine & $50(40-60 \%)$ & Normal & {$[22,23]$} \\
\hline Non-compliers with treatments who recover & $20(10-30 \%)$ & Beta & {$[24,25]$} \\
\hline Sensitivity of mRDT & $95(94.2-96.6 \%)$ & Beta & {$[33]$} \\
\hline Specificity of mRDT & $96(94.8-97.0 \%)$ & Beta & {$[33]$} \\
\hline Sensitivity of clinical diagnosis & $30(20-40 \%)$ & Beta & {$[34]$} \\
\hline Specificity of clinical diagnosis & $90(80-100 \%)$ & Beta & {$[34]$} \\
\hline Adherence to a negative mRDT result ${ }^{\mathrm{b}}$ & $10.5(7.0-14.0 \%)$ & Beta & {$[31,32]$} \\
\hline \multicolumn{4}{|l|}{ Drugs and diagnostic costs (\$US per dose/test) } \\
\hline DhP: Dh $40 \mathrm{mg}$, P $320 \mathrm{mg}$ (“3 $\times 1$ 1” pack) & $0.77(0.56-0.93)$ & Gamma & {$[45]$} \\
\hline AL: A $20 \mathrm{mg}$, L $120 \mathrm{mg}$ (“6 $\times 2$ " pack) & $0.67(0.54-0.84)$ & Gamma & {$[45]$} \\
\hline Quinine $300 \mathrm{mg} /$ tablet & $0.59(0.47-0.70)$ & Gamma & {$[46]$} \\
\hline SP: S $500 \mathrm{mg}$, pyrimethamine $25 \mathrm{mg}$ (“3 31 ” pack) & $0.32(0.25-0.38)$ & Gamma & [46] \\
\hline Quinine injection $300 \mathrm{mg} / \mathrm{mL}$ ( $2 \mathrm{~mL}$ ampoule) & $2.40(1.92-2.87)$ & Gamma & [47] \\
\hline Diazepam injection $5 \mathrm{mg} / \mathrm{mL}$ ( $2 \mathrm{~mL}$ ampoule) & $0.23(0.18-0.27)$ & Gamma & [47] \\
\hline Diclofenac injection $25 \mathrm{mg} / \mathrm{mL}$ ( $3 \mathrm{~mL}$ ampoule) & $0.29(0.23-0.35)$ & Gamma & [47] \\
\hline Dextrose $5 \%(500 \mathrm{~mL}$ bottle $)$ & $3.95(3.16-4.74)$ & Gamma & [47] \\
\hline Ferrous sulphate + folic acid $200+0.25 \mathrm{mg}$ & $0.26(0.21-0.31)$ & Gamma & {$[47]$} \\
\hline Cost of mRDT & $0.45(0.36-0.55)$ & Gamma & {$[30]$} \\
\hline
\end{tabular}

Numbers in the parentheses represent ranges

$A$ artemether, $A C T$ artemisinin-based combination therapy, $A L$ artemether-lumefantrine, $D h$ dihydroartemisinin, $D h P$ dihydroartemisininpiperaquine, $L$ lumefantrine, $m R D T$ Rapid Diagnostic Test for malaria, $P$ piperaquine, $S$ sulfadoxine, $S P$ sulfadoxine-pyrimethamine

${ }^{a}$ As a proportion of dispensed anti-malarial drugs

b Proportion of children who will be treated despite having a negative test result

diagram shown in Fig. 2, which expresses the potential influence of uncertain parameters on budget impact. Cost and the compliance rates for DhP and AL were identified to be the most influential parameters.
Considering that the cost of DhP is the most influential parameter, it was important to show how variation in the cost of DhP causes changes in the budget of drugs and diagnostics, as indicated in Fig. 3. At a cost of about 
Table 2 Annual incremental cost from the perspective of the National Malaria Control Program (\$US)

\begin{tabular}{lllll}
\hline Policy options & mRDT costs & Drug costs & Total cost & Incremental cost \\
\hline AL + quinine & $6,350,032$ & $14,523,023$ & $20,873,055$ & Reference \\
AL + DhP & $6,325,074$ & $14,483,558$ & $20,808,632$ & $-64,423(0.3 \%)$ \\
DhP + AL & $6,232,222$ & $15,356,589$ & $21,588,811$ & $780,180(3.8 \%)$ \\
\hline
\end{tabular}

$A L$ artemether-lumefantrine, $D h P$ dihydroartemisinin-piperaquine, $m R D T$ Rapid Diagnostic Test for malaria

Table 3 Impact on the number of malaria cases and deaths for children aged under 5 years

\begin{tabular}{lllllll}
\hline Policy options & $\begin{array}{l}\text { Uncomplicated } \\
\text { malaria }\end{array}$ & Severe malaria & All cases & $\begin{array}{l}\text { Incremental cases } \\
\text { (all) }\end{array}$ & Number of deaths & Incremental deaths \\
\hline AL + quinine & $7,510,727$ & 173,599 & $7,684,326$ & Reference & 26,973 & Reference \\
AL + DhP & $7,274,872$ & 161,016 & $7,435,888$ & $-248,437(3.2 \%)$ & 25,019 & $-1954(7.2 \%)$ \\
DhP + AL & $6,928,818$ & 142,554 & $7,071,371$ & $-364,517(5.0 \%)$ & 22,151 & $-2868(11.5 \%)$ \\
\hline
\end{tabular}

$A L$ artemether-lumefantrine, $D h P$ dihydroartemisinin-piperaquine

Fig. 2 Incremental Tornado diagram of $\mathrm{DhP}+\mathrm{AL}$ vs. $\mathrm{AL}+\mathrm{DhP} . A L$ artemetherlumefantrine, $D h P$

dihydroartemisininpiperaquine, $E V$ expected value of budget impact

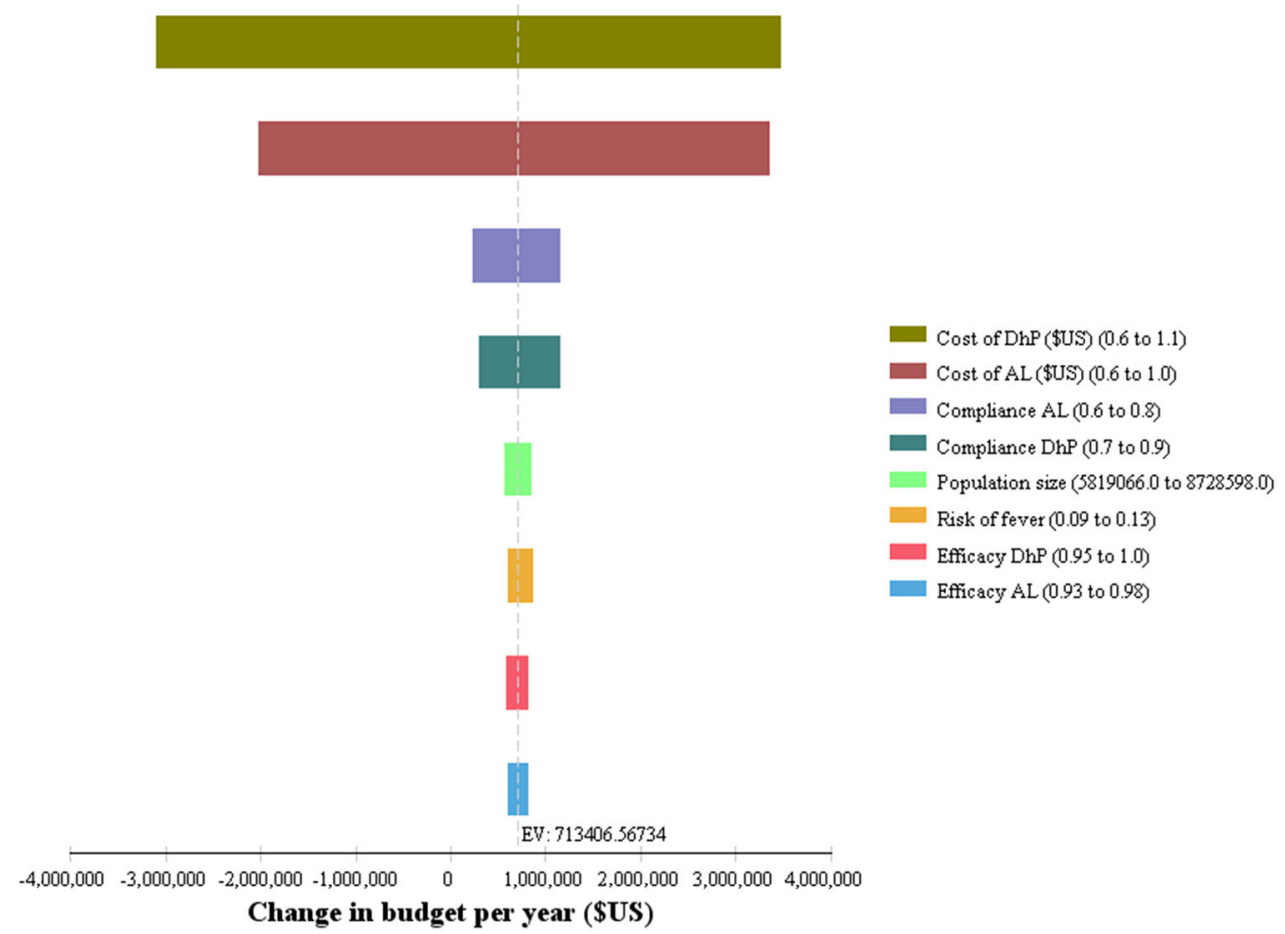

\$US0.84 per dose of DhP, the policy of DhP $+\mathrm{AL}$ is the cheapest option and requires a total budget of about \$US20.7 million annually. It should be noted that this cost includes $20 \%$ program costs; hence, the actual acquisition cost is \$US0.67 per dose. That is to say, for the purpose of cost containment, the additional budget of about \$US780,180 required by the more effective policy of $\mathrm{DhP}+\mathrm{AL}$ may be avoided if DhP could be bought at a price lower than \$US0.67 per dose.
Table 4 shows the budget impact of including hospitalization costs of about \$US76.46 (range \$US60-90) per patient from our previous study [12], with the assumption that all severe cases of malaria will be treated in the public facilities. The model predicts that the policy of AL + DhP will save about \$US934,200 (2.9\%) per year compared with that of $\mathrm{AL}+$ quinine, and that of $\mathrm{DhP}+\mathrm{AL}$ will save about \$US500,342 (1.6\%) per year compared with that of $\mathrm{AL}+\mathrm{DhP}$. Therefore, the policy that uses DhP as 
Fig. 3 Change in total budget of drugs and diagnostics versus variation in the cost of dihydroartemisininpiperaquine. $A L$ artemetherlumefantrine, $D h P$ dihydroartemisinin-piperaquine

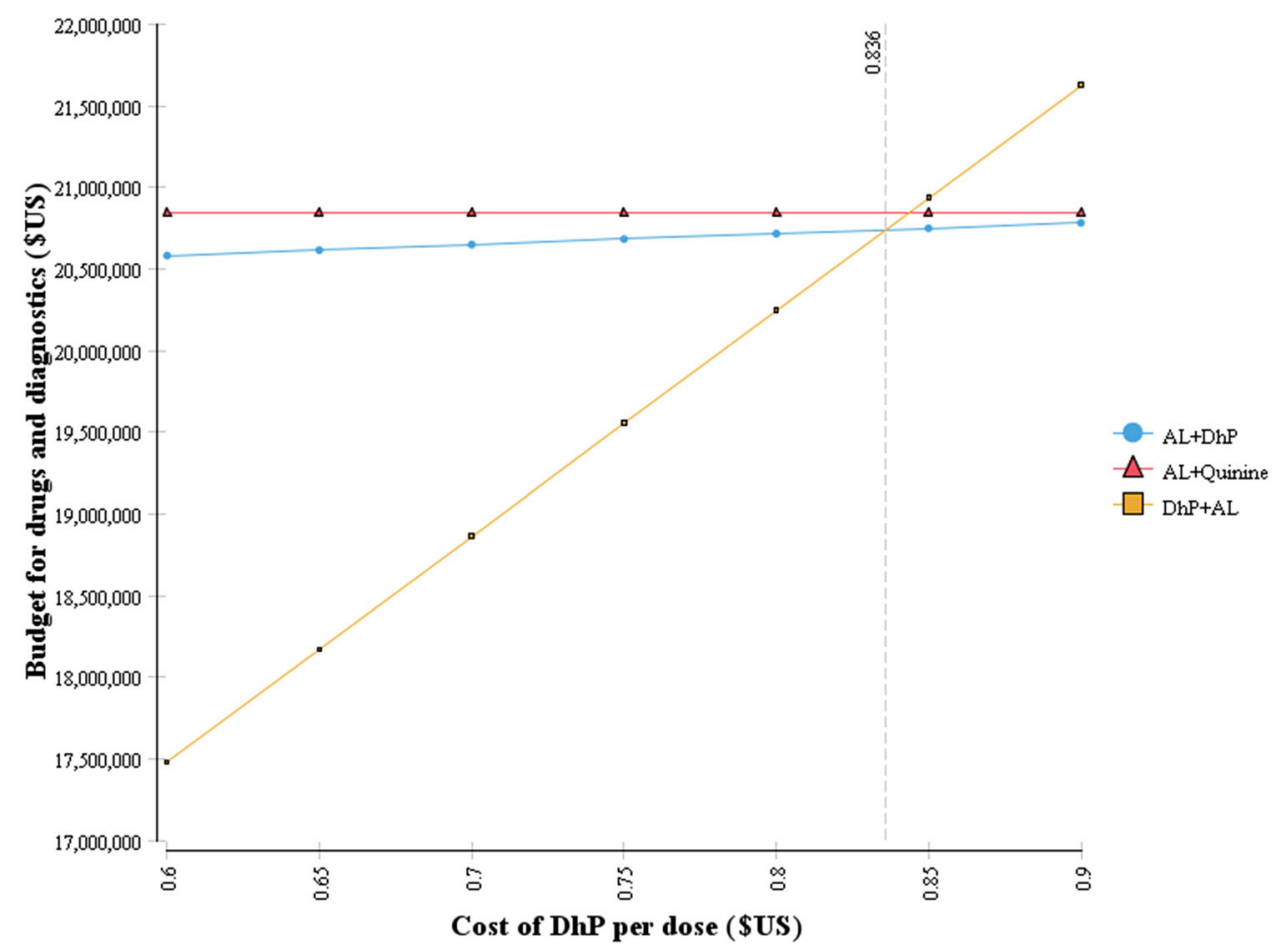

\begin{tabular}{lllll}
\hline Policy options & Drugs and mRDT costs & Hospitalization costs & Total costs & Incremental costs \\
\hline AL + quinine & $20,873,055$ & $11,803,699$ & $32,676,754$ & Reference \\
AL + DhP & $20,808,632$ & $10,933,923$ & $31,742,555$ & $-934,200(2.9 \%)$ \\
DhP + AL & $21,588,811$ & $9,653,401$ & $31,242,212$ & $-500,342(1.6 \%)$ \\
\hline
\end{tabular}

$A L$ artemether-lumefantrine, $D h P$ dihydroartemisinin-piperaquine, $m R D T$ Rapid Diagnostic Test for malaria
Table 4 Budget impact after including hospitalization costs (\$US) (1)

the first-line drug $(\mathrm{DhP}+\mathrm{AL})$ is the most cost-saving treatment policy for uncomplicated malaria when hospitalization costs are taken into consideration.

\section{Discussion}

Malaria is an infectious disease, which consumes a substantial portion of the limited health budgets of sub-Saharan African countries. It is the leading cause of morbidity and mortality among outpatient visits and inpatient admissions in Tanzania. It has been estimated that malaria accounts for about $2 \%$ of the gross domestic product (GDP), which is equivalent to about $20 \%$ of the total health expenditure in Tanzania [52]. This is the first study in Tanzania to estimate the budget impact of introducing DhP to treat uncomplicated malaria in children aged under 5 years either as the first- or second-line drug.

A majority of countries in sub-Saharan Africa are using $\mathrm{AL}$ as the first-line drug and quinine as second-line drug against uncomplicated malaria. Even though ACTs are the preferred choice for the treatment of uncomplicated $P$. falciparum malaria [6], it is understandable that some countries persist with the use of quinine as the second-line drug due to a lack of alternative ACTs. Therefore, the emergence of DhP as a very promising ACT has changed the treatment dynamics of malaria in many countries in recent years. A number of countries have already adopted DhP as the second-line drug and many others are considering doing the same. Mainland Tanzania is one of the countries that recently replaced quinine with DhP as a second-line drug to treat uncomplicated malaria [5].

Several studies have shown that DhP is more cost effective than AL to treat uncomplicated malaria in African countries [8, 11, 12]. However, the arbitrary thresholds of \$US150 per DALY averted or 1-3 times GDP per capita, which are commonly used to categorize interventions as being cost effective or not, may not necessarily reflect the willingness and ability to pay for a new intervention for any particular country $[53,54]$. In reality, a new drug can 
be very cost effective but unaffordable and, for this reason, BIA is increasingly being used to complement cost-effectiveness analyses.

This study found that by considering the costs of drugs and diagnostics only, the policy that uses DhP as the second-line drug $(\mathrm{AL}+\mathrm{DhP})$ to treat uncomplicated malaria in children is slightly cost saving, i.e., $\$$ US64,423 per year, while reducing cases of malaria by about $3 \%$ compared with the policy of $\mathrm{AL}+$ quinine. The model also predicts that the policy that uses $\mathrm{DhP}$ as the first-line drug $(\mathrm{DhP}+\mathrm{AL})$ increases the budget by about $\$ \mathrm{US780,180}$ per year, while reducing the number of malaria cases by a further $5 \%$, compared with $\mathrm{AL}+\mathrm{DhP}$. Pfeil et al. [11] found that the use of DhP as the first-line drug in moderateto-high transmission areas will avert $12 \%$ of malaria cases in children. More recently, Okell et al. [8] estimated a reduction of 10-15\% in high-transmission areas. Our study shows a smaller reduction in malaria cases because we did not consider the longer prophylactic effect of DhP, which reduces the recurrence of malaria $[8,9]$.

Hospitalization costs are an important component of inpatient care costs incurred by healthcare providers to treat severe childhood malaria. The inclusion of hospitalization costs in the sensitivity analysis switched the most cost-saving treatment policy for uncomplicated malaria to DhP + AL, which eventually saves about \$US500,342 per year compared with AL + DhP. Considering that the policy of $\mathrm{DhP}+\mathrm{AL}$ reduces the number of malaria cases by 5 versus $3 \%$ with $\mathrm{AL}+\mathrm{DhP}$, the inclusion of other outpatient and inpatient costs will make $\mathrm{DhP}+\mathrm{AL}$ even more cost saving compared with $\mathrm{AL}+\mathrm{DhP}$.

This study has a number of limitations and, hence, its results must be interpreted with care; more importantly, it should never be used as the sole basis for initiating policy change in Tanzania. Firstly, policy change is a very complex and expensive undertaking [55]. In 2000, it cost the Tanzanian Government about \$US0.8 million to implement the new malaria treatment policy, representing about $4 \%$ of total malaria expenditure and $1 \%$ of total public expenditure on health [56]. Another study estimated the costs of this policy change to be equivalent to $\$$ US0.02 per person [55].

Secondly, care-seeking behavior for diagnosis and treatment of malaria is very complex in sub-Saharan Africa and data may not always be readily available. In the model, a number of structural and parameter assumptions were made in an attempt to replicate how patients move from one type of facility to another in actual practice when seeking care. It is nearly impossible to model this with sufficient accuracy. The model assumptions portray caregivers seeking care from the informal and formal health facilities in a very orderly manner, which rarely happens in reality. Even the diagnosis and treatment practices in public facilities vary from one location to another.

Thirdly, the study assumes that the majority of patients visiting public facilities are diagnosed with mRDTs and all positive cases are prescribed with the recommended first-line drug, with a mean availability of $60 \%$. While we believe that these are reasonable base-case assumptions, several studies have shown that quality of care in public facilities varies greatly and that mRDTs and drugs often completely run out of stock for prolonged periods of time [57, 58]. The availability of a second-line drug was assumed to be restricted in the public facilities, which may not be the case still. Dispensing practice in private drug shops and pharmacies is more complex than the way it is modeled here; hence, the estimated health benefits of the two malaria policies may deviate from the model predictions.

Fourthly, this study ignored the prolonged post-treatment prophylactic effect of $\mathrm{DhP}$, which has more potential to prevent recurrent malaria infections than $\operatorname{AL}[8,9]$. The model shows that an increase in the effectiveness of DhP reduces the number of malaria cases and, hence, treatment costs to the greatest extent when it is used as the first-line drug. The inclusion of a prolonged prophylactic effect would have similar implications, i.e., an increase in health benefits and reduction in treatment costs, which would eventually make the policy of $\mathrm{DhP}+\mathrm{AL}$ even more attractive. Therefore, this study has underestimated the actual benefits of the treatment policy that uses DhP as the first-line drug.

Finally, but equally importantly, rational social planners are concerned not only about reducing healthcare costs but also about broader considerations when distributing scarce healthcare resources, including considerations of efficient and equitable healthcare.

\section{Conclusion}

In accordance with the present model's predictions, the use of $\mathrm{DhP}$ as the second-line drug $(\mathrm{AL}+\mathrm{DhP})$ to treat uncomplicated malaria in children in Tanzania is slightly cost saving. However, the policy that uses DhP as the firstline drug $(\mathrm{DhP}+\mathrm{AL})$ is somewhat more expensive as it consumes an extra $\$$ US780,180 per year, which represents a $3.8 \%$ increase in the budget for drugs and diagnostics, but with more health benefits than AL + DhP. Nevertheless, the use of relatively more expensive drugs such as $\mathrm{DhP}$ as first-line anti-malaria drugs without proper diagnosis should be approached with caution. Otherwise, the opportunity cost of presumptive treatment outweighs the benefits due to over-diagnosis and over-treatment of patients without clinical malaria. 
Acknowledgments The contribution by BR and OFN was supported by the Centre for Intervention Science in Maternal and Child Health (CISMAC; Project Number 223269), which is funded by the Research Council of Norway through its Centres of Excellence Scheme and the University of Bergen (UiB), Norway. We also thank the Priority-Setting and Economics Research Group, and the two anonymous reviewers for their constructive comments on early drafts of the manuscript.

Author contributions ATM, OFN, and BR conceived the study. ATM retrieved the data and performed the analysis. ATM and BR prepared and designed the economic model. ATM prepared the first draft of the manuscript. BR and OFN supervised data analysis and contributed to the manuscript writing. All authors read and approved the final manuscript.

\section{Compliance with Ethical Standards}

This study was funded by the Norwegian State Education Loan Fund (Statens Lånekasse) and the University of Bergen. The funders had no role in the study design, data collection and analysis, decision to publish, or preparation of the manuscript. ATM, OFN, and BR have no conflicts of interest to declare.

Open Access This article is distributed under the terms of the Creative Commons Attribution-NonCommercial 4.0 International License (http://creativecommons.org/licenses/by-nc/4.0/), which permits any noncommercial use, distribution, and reproduction in any medium, provided you give appropriate credit to the original author(s) and the source, provide a link to the Creative Commons license, and indicate if changes were made.

\section{References}

1. WHO. World malaria report 2014. Geneva: World Health Organization; 2014.

2. D'Acremont V, Kilowoko M, Kyungu E, Philipina S, Sangu W, Kahama-Maro J, et al. Beyond malaria-causes of fever in outpatient Tanzanian children. N Engl J Med. 2014;370(9):809-17. doi:10.1056/NEJMoa1214482.

3. Murray CJL, Rosenfeld LC, Lim SS, Andrews KG, Foreman KJ, Haring D, et al. Global malaria mortality between 1980 and 2010: a systematic analysis. Lancet. 2012;379(9814):413-31. doi:10. 1016/s0140-6736(12)60034-8.

4. Ministry of Health and Social Welfare. National guidelines for malaria diagnosis and treatment. Dar es Salaam: National Malaria Control Program; 2006.

5. Ministry of Health and Social Welfare. Standard treatment guidelines and national essential medicine list-Tanzania Mainland. 4th ed. Dar es Salaam: Ministry of Health and Social Welfare; 2013.

6. WHO. Guidelines for the treatment of malaria. 2nd ed. Geneva: World Health Organization; 2010.

7. Zani B, Gathu M, Donegan S, Olliaro PL, Sinclair D. Dihydroartemisinin-piperaquine for treating uncomplicated Plasmodium falciparum malaria. Cochrane Database Syst Rev. 2014;1:CD010927. doi:10.1002/14651858.cd010927.

8. Okell LC, Cairns M, Griffin JT, Ferguson NM, Tarning J, Jagoe $\mathrm{G}$, et al. Contrasting benefits of different artemisinin combination therapies as first-line malaria treatments using model-based costeffectiveness analysis. Nat Commun. 2014;5:5606. doi:10.1038/ ncomms6606.
9. Akpaloo W, Purssell E. Does the use of dihydroartemisininpiperaquine in treating patients with uncomplicated falciparum malaria reduce the risk for recurrent new falciparum infection more than artemether-lumefantrine? Malar Res Treat. 2014;2014:263674. doi:10.1155/2014/263674.

10. Ewing VL, Terlouw DJ, Kapinda A, Pace C, Richards E, Tolhurst $\mathrm{R}$, et al. Perceptions and utilization of the anti-malarials artemether-lumefantrine and dihydroartemisinin-piperaquine in young children in the Chikhwawa District of Malawi: a mixed methods study. Malar J. 2015;14(1):13. doi:10.1186/s12936-0140528-8.

11. Pfeil J, Borrmann S, Tozan Y. Dihydroartemisinin-piperaquine vs. artemether-lumefantrine for first-line treatment of uncomplicated malaria in African children: a cost-effectiveness analysis. PLoS One. 2014;9(4):e95681.

12. Mori AT, Ngalesoni F, Norheim OF, Robberstad B. Cost-effectiveness of dihydroartemisinin-piperaquine compared with artemether-lumefantrine for treating uncomplicated malaria in children at a district hospital in Tanzania. Malar J. 2014;13:363. doi:10.1186/1475-2875-13-363.

13. Sullivan SD, Mauskopf JA, Augustovski F, Jaime Caro J, Lee $\mathrm{KM}$, Minchin M, et al. Budget impact analysis-principles of good practice: report of the ISPOR 2012 Budget Impact Analysis Good Practice II Task Force. Value Health. 2014;17(1):5-14. doi:10. 1016/j.jval.2013.08.2291.

14. United Republic of Tanzania. The 2012 Population and Housing Census: population distribution by age and sex. Dar es-Salaam: National Bureau of Statistics and Office of Chief Government Statistician; 2013.

15. Tanzania Commission for AIDS (TACAIDS), Zanzibar AIDS Commission (ZAC), Nattional Bureau of Statistics (NBS), The Chief Government Statistician (OCGS), ICF International. HIV/ AIDS and Malaria Indicator Survey 2011-2012. Dar es Salaam: TACAIDS, ZAC, NBS, OCGS and ICF International; 2013.

16. National Bureau of Statistics. Tanzania in figures 2012. Dar es Salaam: Ministry of Finance; 2013.

17. National Bureau of Statistics, ICF Macro. Tanzania Demographic and Health Survey 2010. Dar es Salaam: National Bureau of Statistics; 2011.

18. WHO. World health statistics. Geneva: World Health Organization; 2012.

19. Four Artemisinin-Based Combinations (4ABC) Study Group. A head-to-head comparison of four artemisinin-based combinations for treating uncomplicated malaria in African children: a randomized trial. PLoS Med. 2011;8(11):e1001119. doi:10.1371/ journal.pmed.1001119.

20. Bruxvoort K, Goodman C, Kachur SP, Schellenberg D. How patients take malaria treatment: a systematic review of the literature on adherence to antimalarial drugs. PLoS One. 2014;9(1):e84555.

21. Yeka A, Tibenderana J, Achan J, D’Alessandro U, Talisuna AO. Efficacy of quinine, artemether-lumefantrine and dihydroartemisinin-piperaquine as rescue treatment for uncomplicated malaria in Ugandan children. PLoS One. 2013;8(1):e53772.

22. Achan J, Tibenderana JK, Kyabayinze D, Wabwire Mangen F, Kamya MR, Dorsey G, et al. Effectiveness of quinine versus artemether-lumefantrine for treating uncomplicated falciparum malaria in Ugandan children: randomised trial. BMJ. 2009;339:b2763. doi:10.1136/bmj.b2763.

23. Abdulla S, Goodman C, Coleman P, Mubyazi G, Kikumbih N, Okorosobo T. The costs, effects and cost-effectiveness of changing the first line drug for the treatment of malaria in Tanzania. HEFP working paper 01/00. HEFP; 2000. http://r4d.dfid. gov.uk/PDF/Outputs/HealthEcFin_KP/WP01_00.pdf. Accessed 23 Oct 2015. 
24. Coleman PG, Morel C, Shillcutt S, Goodman C, Mills AJ. A threshold analysis of the cost-effectiveness of artemisinin-based combination therapies in sub-saharan Africa. Am J Trop Med Hyg. 2004;71(2 Suppl):196-204.

25. Committee on the Economics of Antimalarial Drugs, Board on Global Health. The cost and cost-effectiveness of antimalarial drugs. In: Arrow KJ, Panosian CB, Gelband H, editors. Saving lives, buying time : economics of malaria drugs in an age of resistance. Washington, DC: National Academies Press; 2004. pp. 61-78.

26. Kahabuka C, Kvale G, Moland KM, Hinderaker SG. Why caretakers bypass Primary Health Care facilities for child care - a case from rural Tanzania. BMC Health Serv Res. 2011;11:315. doi:10. 1186/1472-6963-11-315.

27. Mikkelsen-Lopez I, Tediosi F, Abdallah G, Njozi M, Amuri B, Khatib R, et al. Beyond antimalarial stock-outs: implications of health provider compliance on out-of-pocket expenditure during care-seeking for fever in South East Tanzania. BMC Health Serv Res. 2013;13:444. doi:10.1186/1472-6963-13-444.

28. Kahabuka C, Kvale G, Hinderaker SG. Care-seeking and management of common childhood illnesses in Tanzania-results from the 2010 Demographic and Health Survey. PLoS One. 2013;8(3):e58789. doi:10.1371/journal.pone.0058789.

29. Rao VB, Schellenberg D, Ghani AC. The potential impact of improving appropriate treatment for fever on malaria and nonmalarial febrile illness management in under-5s: a decision-tree modelling approach. PLoS One. 2013;8(7):e69654. doi:10.1371/ journal.pone.0069654.

30. PMI. Malaria operational plan FY 2014. Dar es Salaam: President's Malaria Initiative; 2014.

31. D'Acremont V, Kahama-Maro J, Swai N, Mtasiwa D, Genton B, Lengeler C. Reduction of anti-malarial consumption after rapid diagnostic tests implementation in Dar es Salaam: a before-after and cluster randomized controlled study. Malar J. 2011;10:107. doi:10.1186/1475-2875-10-107.

32. Mubi M, Kakoko D, Ngasala B, Premji Z, Peterson S, Bjorkman $A$, et al. Malaria diagnosis and treatment practices following introduction of rapid diagnostic tests in Kibaha District, Coast Region, Tanzania. Malar J. 2013;12:293. doi:10.1186/1475-287512-293.

33. Reyburn H, Mbakilwa H, Mwangi R, Mwerinde O, Olomi R, Drakeley $\mathrm{C}$, et al. Rapid diagnostic tests compared with malaria microscopy for guiding outpatient treatment of febrile illness in Tanzania: randomised trial. BMJ. 2007;334(7590):403. doi:10. 1136/bmj.39073.496829.AE.

34. Shillcutt S, Morel C, Goodman C, Coleman P, Bell D, Whitty C, et al. Cost-effectiveness of malaria diagnostic methods in subSaharan Africa in an era of combination therapy. Bull World Health Organ. 2008;86(2):101-10. doi:10.2471/blt.07.042259.

35. Mikkelsen-Lopez I, Shango W, Barrington J, Ziegler R, Smith T, deSavigny $\mathrm{D}$. The challenge to avoid anti-malarial medicine stock-outs in an era of funding partners: the case of Tanzania. Malar J. 2014;13:181. doi:10.1186/1475-2875-13-181.

36. Briggs MA, Kalolella A, Bruxvoort K, Wiegand R, Lopez G, Festo $\mathrm{C}$, et al. Prevalence of malaria parasitemia and purchase of artemisinin-based combination therapies (ACTs) among drug shop clients in two regions in Tanzania with ACT subsidies. PLoS One. 2014;9(4):e94074. doi:10.1371/journal.pone.0094074.

37. Mugittu K, Abdulla S, Falk N, Masanja H, Felger I, Mshinda H, et al. Efficacy of sulfadoxine-pyrimethamine in Tanzania after two years as first-line drug for uncomplicated malaria: assessment protocol and implication for treatment policy strategies. Malar J. 2005;4:55. doi:10.1186/1475-2875-4-55.

38. Adeyi O, Atun R. Universal access to malaria medicines: innovation in financing and delivery. Lancet. 2010;376(9755):1869-71. doi:10.1016/s0140-6736(10)61189-0.
39. Mbonye AK, Lal S, Cundill B, Hansen KS, Clarke S, Magnussen $\mathrm{P}$. Treatment of fevers prior to introducing rapid diagnostic tests for malaria in registered drug shops in Uganda. Malar J. 2013;12:131. doi:10.1186/1475-2875-12-131.

40. Morris A, Ward A, Moonen B, Sabot O, Cohen JM. Price subsidies increase the use of private sector ACTs: evidence from a systematic review. Health Policy Plan. 2015;30(3):397-405. doi:10.1093/heapol/czu013.

41. Goodman CA, Coleman PG, Mills AJ. Cost-effectiveness of malaria control in sub-Saharan Africa. Lancet. 1999;354(9176):378-85.

42. de Savigny D, Mayombana C, Mwageni E, Masanja H, Minhaj A, Mkilindi Y, et al. Care-seeking patterns for fatal malaria in Tanzania. Malar J. 2004;3:27. doi:10.1186/1475-2875-3-27.

43. Lubell Y, Staedke SG, Greenwood BM, Kamya MR, Molyneux M, Newton PN, et al. Likely health outcomes for untreated acute febrile illness in the tropics in decision and economic models; a Delphi survey. PLoS One. 2011;6(2):e17439. doi:10.1371/ journal.pone.0017439.

44. Dondorp AM, Fanello CI, Hendriksen ICE, Gomes E, Seni A, Chhaganlal KD, et al. Artesunate versus quinine in the treatment of severe falciparum malaria in African children (AQUAMAT): an open-label, randomised trial. Lancet. 2010;376(9753):1647-57. doi:10.1016/s0140-6736(10)61924-1.

45. The Global Fund. ACT Prices under the Affordable Medicines Facility-malaria: update of 22 May 2013. Fact sheet. Geneva: The Global Fund; 2013.

46. Management Science for Health. International drug price indicator guide. 2013th ed. Arlington: Management Science for Health, Inc.; 2014.

47. Medical Stores Department. Price catalogue of essential medicines, diagnostics and hospital supplies-2014/2015. Dar es Salaam: Medical Stores Department; 2014.

48. Bank of Tanzania. Monthly economic review: October 2014. Dar es Salaam: Bank of Tanzania; 2014.

49. Lubell Y, Riewpaiboon A, Dondorp AM, von Seidlein L, Mokuolu OA, Nansumba M, et al. Cost-effectiveness of parenteral artesunate for treating children with severe malaria in subSaharan Africa. Bull World Health Organ. 2011;89(7):504-12. doi:10.2471/BLT.11.085878.

50. WHO. World malaria report 2013. Geneva: World Health Organization; 2013.

51. Lozano R, Wang H, Foreman KJ, Rajaratnam JK, Naghavi M, Marcus JR, et al. Progress towards Millennium Development Goals 4 and 5 on maternal and child mortality: an updated systematic analysis. Lancet. 2011;378(9797):1139-65. doi:10.1016/ s0140-6736(11)61337-8.

52. Department of Policy and Planning, Ministry of Health and Social Welfare, United Republic of Tanzania. Tanzania national health accounts year 2010: with sub-accounts for HIV and AIDS, malaria, reproductive, and child health. Dar es Salaam: Department of Policy and Planning, Ministry of Health and Social Welfare, United Republic of Tanzania; 2012.

53. Shillcutt SD, Walker DG, Goodman CA, Mills AJ. Cost-effectiveness in low- and middle-income countries: a review of the debates surrounding decision rules. Pharmacoeconomics. 2009;27(11):903-17.

54. Woods B, Revill P, Sculpher M, Claxton K. Country-level costeffectiveness thresholds: initial estimates and the need for further research. CHE research paper 109. York: Centre for Health Economics, University of York; 2015.

55. Mulligan JA, Mandike R, Palmer N, Williams H, Abdulla S, Bloland $\mathrm{P}$, et al. The costs of changing national policy: lessons from malaria treatment policy guidelines in Tanzania. Trop Med Int Health. 2006;11(4):452-61. doi:10.1111/j.1365-3156.2006. 01590.x. 
56. Jowett M, Miller N, Mnzava N. Malaria expenditure analysis: Tanzania Case Study. Dar es Salaam: DFID-EA (Tanzania) and the Roll Back Malaria Initiative; 2000.

57. Kahabuka C, Kvale G, Hinderaker SG. Factors associated with severe disease from malaria, pneumonia and diarrhea among children in rural Tanzania - a hospital-based cross-sectional study. BMC Infect Dis. 2012;12:219. doi:10.1186/1471-2334-12-219.
58. Kapologwe N, Mori AT, Chilunda F, Meshack M, Kalolo A, Wiedenmayer K. Reforming supportive supervision of medicines management with an audit tool in primary health care facilities: a case study of Bahi District. Tanzania. Int $\mathrm{J}$ Pharm. 2014;4(4):108-14. 